\title{
Notas / Notes \\ Las especies de Hyalopterus Koch, 1854 (Hemiptera, Aphididae), pulgones harinosos de los frutales de hueso, en España peninsular e Islas Baleares
}

\author{
Víctor Moreno-González ${ }^{1}$ \& Juan M. Nieto Nafría ${ }^{2}$ \\ 'Departamento de Biodiversidad y Gestión Ambiental, área de Zoología. Universidad de León. 24071 León (España); \\ vmoreg00@estudiantes.unileon.es; ORCID ID: https://orcid.org/0000-0003-0094-1559 \\ ${ }^{2}$ Departamento de Biodiversidad y Gestión Ambiental, área de Zoología. Universidad de León. 24071 León (España); \\ jmnien@unileon.es; ORCID ID: http://orcid.org/0000-0001-6714-2415
}

\section{RESUMEN}

Se ha estudiado la distribución de las especies del género Hyalopterus Koch, 1854 (Aphidinae, Aphidini, Rhopalosiphina) en España peninsular e Islas Baleares mediante la revisión de las citas bibliográficas, del material depositado en la colección de la Universidad de León y de las fotografías almacenadas en Insectarium virtual. Se actualiza la distribución de las especies y aportan citas en nuevas provincias. Además, se aporta una clave de identificación para las hembras vivíparas ápteras de las especies de Hyalopterus.

Palabras clave: España; Hyalopterus; Rhopalosiphina.

\section{ABSTRACT}

Species of Hyalopterus (Hemiptera, Aphididae), stone fruit mealy aphids, in Spanish mainland and Balearic Islands

Distribution of the genus Hyalopterus Koch, 1854 (Aphidinae, Aphidini, Rhopalosiphina) in Spanish mainland and Balearic Islands has been studied by the review of the bibliographic records, the material deposited in Universidad de León collection and the photographs stored in Insectarium virtual. The distribution of Hyalopterus species is updated and new records are reported. An identification key for the viviparous females of Hyalopterus species is also presented.

Key words: Hyalopterus; Rhopalosiphina; Spain.

Recibido/Received: 10/01/2018; Aceptado/Accepted: 21/09/2018; Publicado en línea/Published online: 19/10/2018

Cómo citar este artículo/Citation: Moreno-González, V. \& Nieto Nafría, J. M. 2018. Las especies de Hyalopterus Koch, 1854 (Hemiptera, Aphididae), pulgones harinosos de los frutales de hueso, en España peninsular e Islas Baleares. Graellsia, 74(2): e078. https://doi.org/10.3989/graellsia.2018.v74.197

Copyright: (C) 2018 SAM \& CSIC. This is an open-access article distributed under the terms of the Creative Commons Attribution 4.0 International (CC BY 4.0) License.

Entre los pulgones (Hemiptera, Aphididae) que afectan a los frutales de hueso (varias especies del género Prunus [Rosaceae]) se encuentran las especies del género Hyalopterus Koch, 1854 (Aphidinae, Aphidini, Rhopalosiphina). En una reciente revisión taxonómica-nomenclatural (Favret et al., 2017) se ha admitido que el género incluye tres especies subjetivamente válidas: $H$. amygdali (Blanchard, 1840), $H$. arundiniformis Ghulamullah, 1942 y $H$. pruni (Geoffroy, 1762), y se ha reconocido que H. mimulus
Börner, 1950 y H. persikonus Miller, Lozier \& Foottit, 2008 son sinónimas de $H$. arundiniformis, y que H. spinarum (Hartig, 1841) lo es de H. pruni. El género Hyalopterus es de origen paleártico occidental, con citas de sus especies en territorios fuera de Europa y del occidente de Asia (Blackman \& Eastop, 2017) como consecuencia de la actividad antrópica y porque unas u otras de sus especies se desarrollan sobre albaricoqueros, almendros, cerezos, ciruelos, melocotoneros y otras especies cultivadas del género Prunus. 
En estos momentos está admitido que las tres especies (Rakauskas et al., 2013; Blackman \& Eastop, 2017) son dioicas holocíclicas. Por ser holocíclicas presentan una generación anfigónica con hembras ovíparas ápteras que en otoño serán fecundadas por machos alados y pondrán los huevos de invierno, y múltiples generaciones de hembras partenogenéticas y vivíparas que se suceden desde la primavera hasta el otoño, con ápteras y aladas. Por ser dioicas están obligadas a cambiar de hospedador dos veces durante el ciclo anual. En el hospedador primario (plantas de especies de Prunus) nacen las hembras ovíparas - de vivíparas aladas migrantes, denominadas ginóparasson puestos los huevos de invierno, de los que nacen las fundadoras, las cuales dan origen a la primera generación de las varias de fundatrígenas, en la última de las cuales hay hembras aladas que migran al hospedador secundario (especies de los géneros Phragmites, Arundo u otros próximos), en el que transcurre la vida de hembras vivíparas de varias generaciones, ápteras o aladas (virginógenas), y en el que nacen los machos y las ginóparas antes mencionadas, quienes realizan el vuelo de regreso al hospedador primario. Sin embargo, subsisten dudas sobre la posibilidad de que alguna de las especies o de sus poblaciones hayan suprimido la migración, habiendo pasado a ser paramonoicas en el hospedador primario, en el que se mantendrían todo el año, o que incluso hayan suprimido la generación anfigónica, habiendo pasado a ser anholocíclicas (Blackman \& Eastop, 2017).

García Prieto et al. (2004) reseñaron la distribución conocida en la península Ibérica e Islas Baleares de $H$. amygdali y de $H$. pruni. Pérez Hidalgo \& Mier Durante (2005) reiteraron esos datos y expusieron los caracteres del género y de ambas especies. La primera cita de $H$. arundiniformis en España y en concreto de la provincia de Alicante se debe a Lozier et al. (2008) bajo el nombre de $H$. persikonus. En Insectarium Virtual (Biodiversidad Virtual, 2017) se exponen fotografías de especímenes del género tomadas en muchas provincias españolas, algunas con identificación específica y la mayoría sólo con identificación a nivel genérico; de algunas de esas provincias no hay citas en publicaciones tradicionales (Fig. 1, provincias marcadas con " $F$ ").

Con el fin de mejorar el conocimiento de la distribución de las especies de este género, así como de su bionomía y de su peligrosidad para los cultivos de frutales de hueso en el territorio español, se plantearon dos objetivos: 1) informar de la auténtica distribución conocida en España peninsular e Islas Baleares del género y de sus tres especies, y 2) facilitar caracteres que permitan a personas no especializadas en la taxonomía afídica efectuar la identificación - a nivel de género o de especie- de especímenes de estas especies mediante caracteres biológicos y morfológicos, tanto macroscópicos como microscópicos.

Para alcanzar esos objetivos se revisaron críticamente los artículos científicos en los que se cita alguna de las especies del género en España [37 desde el de Mimeur (1936) hasta el de Lozier et al. (2008)], se evaluaron los caracteres distintivos ofrecidos por diferentes autores (los ya mencionados Pérez Hidalgo \& Mier Durante, 2005; Lozier et al., 2008; Rakauskas et al. 2013; Blackman \& Eastop, 2017), se estudiaron microscópicamente los ejemplares de la colección de preparaciones de pulgones de la Universidad de León y se revisaron las fotografías depositadas en Insectarium Virtual (Biodiversidad Virtual, 2017) atendiendo al hospedador.

Se puede asegurar que han sido recogidos o en su caso fotografiados especímenes pertenecientes al género Hyalopterus en las provincias sombreadas en la Figura $1 \mathrm{~A}$, sin que se pueda identificar la especie de los correspondientes a las provincias de Álava, Ávila, Gerona, Huelva, Málaga, Orense y Toledo (con punto de interrogación en la Figura 1), y que se han citado, con el mismo nombre o con otro por inicial identificación incorrecta, cada una de las tres especies en las provincias que se relacionan a continuación:

Hyalopterus amygdali en Alicante, Almería, Barcelona, Cáceres, Castellón, Cuenca, Granada, Islas Baleares, Jaén, Madrid, Murcia, Palencia, Salamanca, Sevilla, Soria, Tarragona, Valencia y Zamora (Fig. 1B).

Hyalopterus arundiniformis en Alicante, Cádiz, Cantabria, Castellón, Córdoba, Granada Murcia, Salamanca, Segovia, Sevilla, Valencia y Zamora (Fig. 1C).

Hyalopterus pruni en Alicante, Almería, Castellón, Huesca, León, Lérida, Madrid, Navarra, Soria, Teruel, Valencia y Zamora (Fig.1D).

Los mapas muestran: 1) que es muy posible que las tres especies se encuentren en todas las provincias peninsulares y en Baleares; y 2) que la cita de una u otra de las especies en las provincias dónde se haya producido se debe a la oportunidad de la captura. Es muy posible que la distribución potencial de estas especies esté condicionada a la presencia de plantas que puedan utilizar como hospedadores secundarios (Arundo o Phragmites) o como hospedadores primarios, sean estos últimos frutales de hueso ( $P$. armeniaca, $P$. avium, $P$. cerasus, $P$. domestica, $P$. dulcis, $P$. persica o $P$. salicina) o especies de uso ornamental, como $P$. cerasifera, o silvestres como $P$. spinosa, endrino, y $P$. mahaleb, cerezo de santa Lucía.

El género es fácilmente reconocible a simple vista por ser pulgones: (a) relativamente grandes (1,5 a casi $3 \mathrm{~mm}$ de longitud corporal), (b) de color verde o menos frecuentemente rosado a granate - las partenogenéticas aladas tienen cabeza y tórax pardos- pero recubiertos de una pulverulencia blanquecina, que a veces es muy abundante y que puede permanecer en las hojas una vez que la colonia ha desaparecido, y (c) que suelen formar grupos compactos normalmente en el envés de las hojas, pero a veces también sobre el haz y en peciolos foliares y brotes.

La identificación de las ápteras mediante caracteres morfológicos no se puede realizar a simple vista y la 


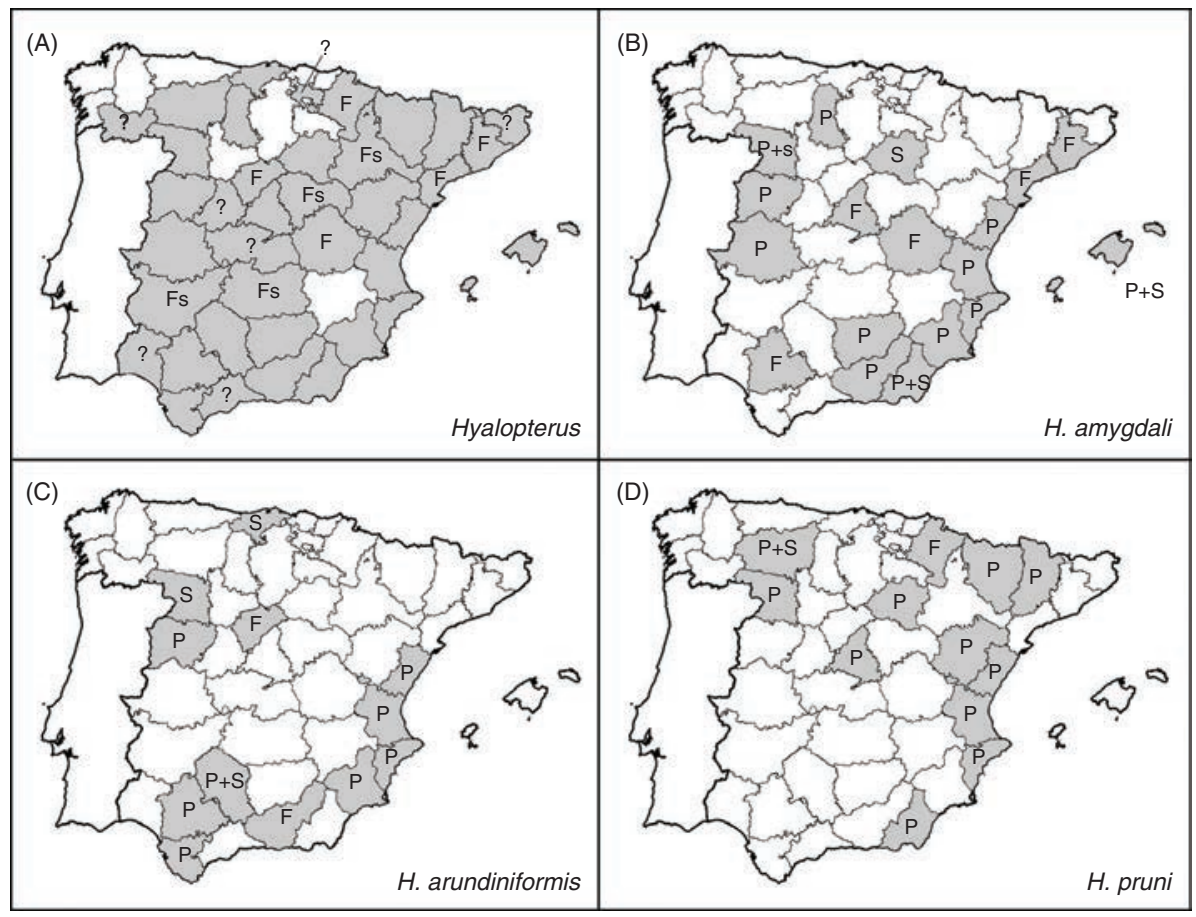

Fig. 1.- Mapas de distribución del género Hyalopterus (A) y de sus especies, H. amygdali(B), H. arundiniformis (C) y H. pruni (D). Las provincias con presencia del género o las especies aparecen sombreadas. Mapa A: ?: cita bibliográfica sin identificación a nivel especifico; F: cita de Insectarium virtual; FS: cita de Insectarium virtual sobre el hospedador secundario y sin identificación específica. Mapas B-D: P: citas sobre el hospedador primario; S: citas sobre el hospedador secundario, F: cita de Insectarium virtual sobre el hospedador primario.

Fig. 1.- Distribution maps of the genera Hyalopterus (A) and its species, H. amygdali (B), H. arudiniformis (C) and $H$. pruni (D). The provinces where both, the genera or the species, are recorded, are shaded. Map A: ?: bibliographic record without species identification; F: Insectarium Virtual record; FS: Insectarium Virtual record on the secondary host, without species identification. Maps B-D: P: primary host records; S: secondary host records; F: Insectarium Virtual records on primary host.

de las aladas se tiene por imposible en estos momentos si no se conoce la planta hospedadora primaria en la que viven o de la que procedan (Lozier et al., 2008; Rakauskas et al., 2013; Blackman \& Eastop, 2017).

Una identificación específica presuntiva se puede realizar en muchos casos recurriendo a la especie de Prunus sobre la que se estén desarrollando los ejemplares en estudio, porque según los datos de los que se dispone: $H$. amygdali puede vivir solamente sobre $P$. armeniaca y $P$. dulcis, $H$. arundiniformis puede hacerlo solamente sobre $P$. persica y $P$. armeniaca, y por último $H$. pruni se puede mantener sobre $P$. armeniaca, $P$. avium, $P$. cerasifera, $P$. cerasus, $P$. domestica, $P$. salicina y las especies silvestres mencionadas. En consecuencia, es posible inferir la especie de pulgón a partir de la planta, excepto en el caso de los especímenes que viven sobre albaricoqueros (P. armeniaca).

Comprobar la identificación hecha por la planta hospedadora o dilucidarla si los ejemplares se desarrollaban sobre albaricoquero ( $P$. armeniaca) o sobre carrizos o cañas (hospedadores secundarios), exige el estudio de hembras vivíparas ápteras montadas entre porta y cubre (para el procedimiento de montaje véase Nieto Nafría \& Mier Durante, 1998). Una identificación aceptablemente segura se puede alcanzar por la distinta forma y longitud de las setas dorsales de la cabeza (Fig. 2); identificación que en caso de necesidad se puede corroborar usando las claves más complejas de Lozier et al. (2008) y de Rakauskas et al. (2013).

\section{Clave para la identificación de hembras vivíparas ápteras de Hyalopterus}

1 - Longitud de las setas dorsales medias de la cabeza $0,14-0,30$ veces la distancia entre sus bases (Fig. 2A). Setas anteriores de la cabeza robustas. [Sobre P. dulcis y P. armeniaca, Ph. australis o A. donax] ......... Hyalopterus amygdali (Blanchard, 1840)

- Longitud de las setas dorsales medias de la cabeza más de 0,30 veces la distancia entre sus bases. Setas anteriores de la cabeza filiformes (en todo caso menos robustas que las de $H$. amygadali). [Sobre P. domestica, P. persica o P. armeniaca, Ph. australis o A. donax ] ........ 2

2 - Longitud de las setas dorsales medias de la cabeza 0,30-0,40 veces la distancia entre sus bases (Fig. 2C). [Sobre varias especies de Prunus, incluyendo $P$. domestica, $P$. salicina y $P$. armeniaca, pero no sobre P. dulcis, ni P. persica, o sobre Ph. australis o A. donax ] ............ Hyalopterus pruni (Geoffroy, 1762) - Longitud de las setas dorsales medias de la cabeza 0,470,70 veces la distancia entre sus bases (Fig. 2B). [Sobre $P$. persica y $P$. armeniaca, $P h$. australis o A. donax] ... Hyalopterus arundiniformis Ghulamullah, 1942 


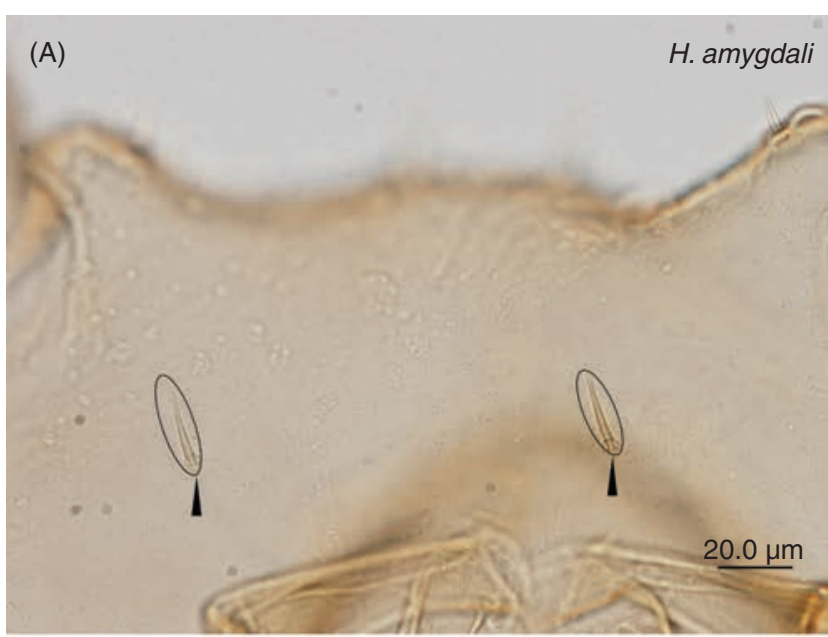

(B)

H. arundiniformis
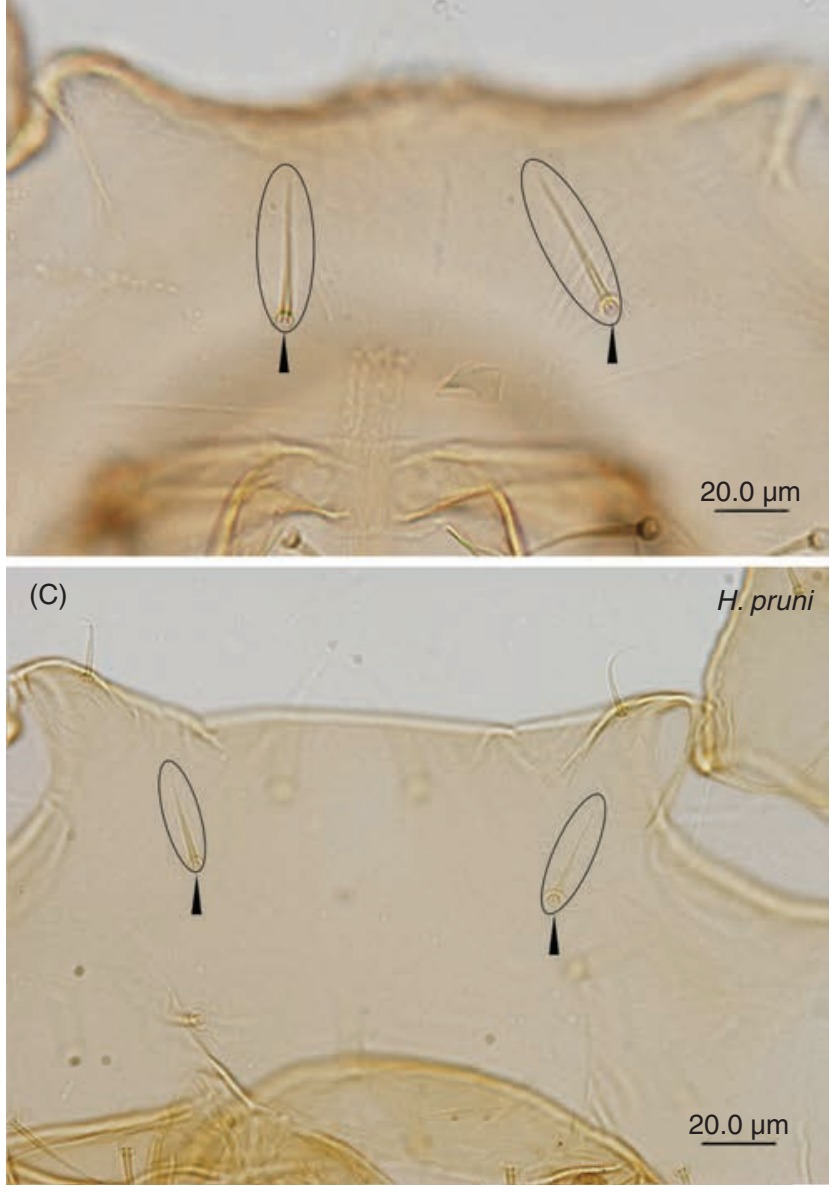

Fig. 2.- Detalle de las setas dorsales medias de la cabeza de $H$. amygdali $(A), H$. arundiniformis $(B)$ y $H$. pruni $(C)$. Las flechas y las elipses señalan las setas dorsales medias de la cabeza. Las fotografías fueron tomadas por S.I. López Ciruelos.

Fig. 2.- Detail of medium dorsal head setae of $H$. amygdali (A), $H$. arundiniformis $(\mathrm{B})$ and $H$. pruni $(\mathrm{C})$. The arrowheads and the ellipses mark the median dorsal head setae. Photographs were taken by S.I. López Ciruelos.

\section{Agradecimientos}

Nuestro agradecimiento a Sara Isabel López Ciruelos por las fotografías de los ejemplares de Hyalopterus y al equipo de Biodiversidad Virtual por facilitarnos los datos de localidad de las fotografías almacenadas en Insectarium Virtual.

\section{Referencias}

Blackman, R.L. \& Eastop, V.F., 2017. Aphids on the world plants. An online identification and information guide. Disponible en: http://www.aphidsonworldsplants.info. Accedido: 1 de diciembre de 2017

Biodiversidad Virtual, 2017. Invertebrados. Insectarium Virtual. Disponible en: http://www.biodiversidadvirtual.org/insectarium/. Accedido: 15 de noviembre de 2017

Favret, C., Meshram, N.M., Miller, G.L., Nieto Nafría, J.M. \& Stekolshchikov, A.V., 2017. The mealy plum aphid and its congeners: a synonymic revision of the Prunus-infesting aphid genus Hyalopterus (Hemiptera: Aphididae). Proceedings of the Entomological Society of Washington, 119(4): 565-574. https://doi. org/10.4289/0013-8797.119.4.565

García Prieto, F., Pérez Hidalgo, N., Mier Durante, M.P. \& Nieto Nafría, J.M., 2004. Updated check-list of IberianBalearic Aphidini (Hemiptera, Aphididae). Graellsia, 60: 197-214. https://doi.org/10.3989/graellsia.2004. v60.i2.215

Lozier, J.D., Foottit, R.G., Miller, G.L., Mills, N.J. \& Roderick, G.K., 2008. Molecular and morphological evaluation of the aphid genus Hyalopterus Koch (Insecta: Hemiptera: Aphididae), with a description of a new species. Zootaxa, 1688: 1-19.

Mimeur, J.M., 1936. Aphididae (Hem.) d'Espagne. Boletín de la Sociedad Entomológica de España, 27: 33-40.

Nieto Nafría, J.M. \& Mier Durante, M.P., 1998. Hemiptera, Aphididae I. In: Ramos, M.A. et al. (Eds.). Fauna Ibérica, vol. 11. Museo Nacional de Ciencias Naturales, CSIC. Madrid. 424 pp.

Pérez Hidalgo, N. \& Mier Durante, M.P., 2005. Género Hyalopterus. In: Nieto Nafría, J.M., Mier Durante, M.P., García Prieto, F. \& Pérez Hidalgo, N. Hemiptera, Aphididae III. In: Ramos, M.A. et al. (Eds.). Fauna Ibérica, vol. 28. Museo Nacional de Ciencias Naturales, CSIC. Madrid: 208-213.

Rakauskas, R., Havelka, J. \& Zaremba, A., 2013. Mitochondrial COI and morphological specificity of the mealy aphids (Hyalopterus spp.) collected from different hosts in Europe (Hemiptera, Aphididae). ZooKeys, 319: 255-267. https://doi.org/10.3897/ zookeys.319.4251 